\title{
Spatially Nonlinear Interdependence of Alpha-Oscillatory Neural Networks under Chan Meditation
}

\author{
Pei-Chen Lo ${ }^{1}$ and Chih-Hao Chang ${ }^{2}$ \\ ${ }^{1}$ Department of Electrical Engineering, Institute of Electrical and Control Engineering, National Chiao Tung University, \\ Hsinchu 30010, Taiwan \\ ${ }^{2}$ Institute of Electrical and Control Engineering, National Chiao Tung University, Hsinchu 30010, Taiwan \\ Correspondence should be addressed to Pei-Chen Lo; pclo@faculty.nctu.edu.tw
}

Received 9 June 2013; Revised 12 October 2013; Accepted 13 November 2013

Academic Editor: Hector Tsang

Copyright (C) 2013 P.-C. Lo and C.-H. Chang. This is an open access article distributed under the Creative Commons Attribution License, which permits unrestricted use, distribution, and reproduction in any medium, provided the original work is properly cited.

\begin{abstract}
This paper reports the results of our investigation of the effects of Chan meditation on brain electrophysiological behaviors from the viewpoint of spatially nonlinear interdependence among regional neural networks. Particular emphasis is laid on the alpha-dominated EEG (electroencephalograph). Continuous-time wavelet transform was adopted to detect the epochs containing substantial alpha activities. Nonlinear interdependence quantified by similarity index $S(\mathbf{X} \mid \mathbf{Y})$, the influence of source signal $\mathbf{Y}$ on sink signal $\mathbf{X}$, was applied to the nonlinear dynamical model in phase space reconstructed from multichannel EEG. Experimental group involved ten experienced Chan-Meditation practitioners, while control group included ten healthy subjects within the same age range, yet, without any meditation experience. Nonlinear interdependence among various cortical regions was explored for five local neural-network regions, frontal, posterior, right-temporal, left-temporal, and central regions. In the experimental group, the inter-regional interaction was evaluated for the brain dynamics under three different stages, at rest (stage R, pre-meditation background recording), in Chan meditation (stage $\mathrm{M}$ ), and the unique Chakra-focusing practice (stage C). Experimental group exhibits stronger interactions among various local neural networks at stages $\mathrm{M}$ and $\mathrm{C}$ compared with those at stage $\mathrm{R}$. The intergroup comparison demonstrates that Chan-meditation brain possesses better cortical inter-regional interactions than the resting brain of control group.
\end{abstract}

\section{Introduction}

For several decades, scientific exploration has corroborated the effectiveness of meditation practice on health promotion. Particular evidence includes the improvement of cardiovascular functions, immunity, and hormone-level regulation. In addition, meditation makes positive changes in the brain and mind, including the positive emotional states, better stress manipulation, enhanced mindful attention, noticeable anxiety reduction, and depression relief, [1-7].

During the past decades, a number of meditation techniques have been developed and practiced all over the world. Although with somewhat different practicing scheme, almost all the practices are aimed to better manipulate the mind, brain function, and physical state of practitioners through mindfulness concentration and respiratory regulation. For many centuries, eastern religious and secular groups, such as the Buddhists, Taoist traditionalists, and the Indian Yogis, have been practicing meditation in order to achieve certain physical, mental, and spiritual realm. Meditation is a unique state of transcendental consciousness beyond the normal mind and mental process. Meditation may induce a series of integrated physiological changes. Among the diverse types of meditation, most practitioners are able to experience complete relaxation and the so-called tranquil awareness.

Although individuals in the East have been practicing various forms of meditation throughout history, scientific study of meditation did not begin until it became popular in the West. In recent years, meditation having been extended to complementary medical practices further motivated scientific studies with the focus of physiological alterations induced by the process $[8,9]$. Increasing reports of meditation 
benefits further draw attention of researchers to the assessment of meditation in different indications. The research for physical and psychological correlation of meditation has been concentrated mostly on Yoga and transcendental meditation (TM) from India, Japanese Zen, and Tibetan Buddhism $[10,11]$. Up to the present, little has yet been disclosed regarding the phenomena of Chan-Buddhist meditation (or simply "Chan meditation"). In the past decade, orthodox Chan meditation, as an unconventional therapy, has proved to be efficacious for many chronic diseases, infections, and even some malignant tumors. Consequently, more people began to practice orthodox Chan meditation in Taiwan. Accumulation of the effective evidences and health benefits of Chan meditation aroused our attention to the physiological investigation on the Chan-Buddhist disciples.

Since meditation process involves different states of mental activities and consciousness, EEG (electroencephalograph) thus became our major focus for exploring the human life system under Chan meditation. EEG applications in clinic and medical centers have become favorable since the 1970s because of its advantages of economy, safety, and convenience. Most of all, more scientific evidences of EEG variations have been disclosed in a number of different physiological, pathological, conscious and mental states in accordance with the various temporal, spectral, and spectral EEG characteristics. Although with the rapid progress in sophisticated medical imaging technologies, EEG still plays an important and irreplaceable role in long-term monitoring of brain functions exhibited as the lump variations of electrical activities. New findings have been continuously observed and reported [12-15]. As normally characterized by frequency, the EEG patterns are conveniently classified into five frequency ranges including delta $(\Delta$, below $4 \mathrm{~Hz})$, theta $(\theta, 4-8 \mathrm{~Hz})$, alpha $(\alpha, 8-13 \mathrm{~Hz})$, beta $(\beta, 13-30 \mathrm{~Hz})$, and gamma $(\gamma, 30-70 \mathrm{~Hz})$. Earlier paper [16], based on EEG spectral power and coherence estimates, reported the brain regions involved in meditative states as the selective associations of theta and alpha oscillating networks activity with states of internalized attention and positive emotional experience. According to our preliminary results, differentiation in frontal/occipital alpha activities plays a key role in comparing EEG between Chan-meditation practitioners (Appendix) and normal, healthy non-meditating subjects.

To explore the spatial interactions among brain local neural networks under alpha-rhythmic oscillation, methods developed in nonlinear dynamical theory become more versatile and favorable $[17,18]$. The interactions among separate brain regions play a significant role in understanding the neurophysiological behavior of human brain. Accordingly, multivariate time series analysis based on nonlinear dynamical modeling becomes much appealing to investigate the important mechanism by which specialized cortical and subcortical regions integrate their activities into different functions and different spatial scales [19-23]. In recent studies, brain dynamics can be conceived as a large ensemble of coupled nonlinear dynamical subsystems. We have focused on investigating the nonlinear, chaotic characteristics of Chan-meditation EEG during the past decade, based on nonlinear deterministic modeling of brain dynamics [14,
24]. Significant nonlinear synchronization has been detected between the macroscopic scale of EEG channels. Thus, various types of synchronization based on the concepts of nonlinear dynamical systems theory have previously been proposed as a more powerful mechanism than narrow band frequency synchronization (e.g., coherence function) for achieving integrative neural processing. This type of "nonlinear coupling" allows studying nonlinear interdependence between multichannel recording sites and represents an alternative to the coherence function which addresses all of these limitations simultaneously [24-32]. As a consequence, this study aims to probe into the $\alpha$-wave nonlinear interdependence behaviors among multichannel electroencephalograph (EEG) signals collected from the orthodox Chan-Buddhist practitioners (experimental group) and normal, healthy subjects (control group).

\section{Material and Methods}

2.1. Voluntary Subjects and Procedures. This study involved two groups of subjects, the experimental group including 10 volunteers with an average of 5.8-year Heart-Sealing Chanmeditation experience and the control group including 10 volunteers without any meditation experience. Seven men and three women were in either group. In the experimental group the average age was 28 years, while in the control group the average age was 23 years. Heart-Sealing Chanmeditation practitioners participated in one 90 -minute group meditation session every week and practiced approximately 30-minute individual meditation on a daily basis. HeartSealing Chan meditation has been the only orthodox way of inheriting the lineage of Chan sect. The core essence of orthodox Chan-meditation practice is to transcend the physiological (fifth), mental (sixth), subconscious (seventh), and Alaya (eighth) states of consciousness and finally attain the realm of true self characterized by the pure golden light with eternal wisdom (Appendix). All the consciousnesstranscending preprocesses inside can be completed for a well experienced orthodox Chan-meditation practitioner who has been able to spontaneously activate ten Chakras in our body [33]. These ten Chakras are important energy spots for altering states of consciousness by converting our physically and mentally dominant characteristics to a particular state of detachment. Accordingly, novice practitioners put lots of effort into the practice from Chakra focusing, Chakra perception, up to Chakra sealing. In the beginning stage of Chakra focusing, practitioners may practice the special brain-drilling technique to reduce all the wandering thoughts abiding in the brain. The brain-drilling technique involves, firstly, focusing alternately on frontal and posterior regions of the brain with mindfulness attention and, next, focusing alternately on left and right regions and perceiving the interconnections between two regions.

In the experiment, we conducted overall 50-minute recording of EEG signals for both groups. The EEG signals were recorded by the 30-channel, common-reference (linkedmastoid MS1-MS2) electrode montage based on the international 10-20 system. Figure 1 illustrates the EEG recording montage of the 30 electrode locations. The protocol designed 


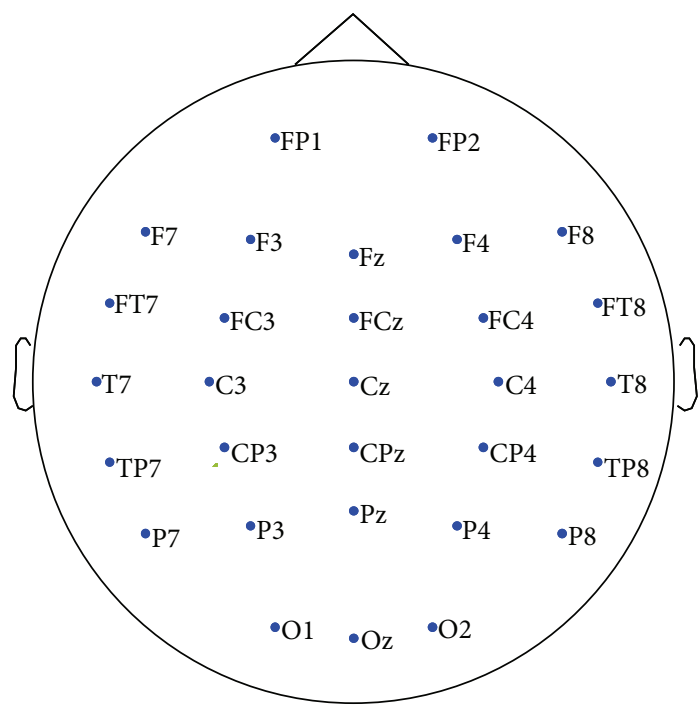

FIGURE 1: EEG electrode locations of the 30-channel recording montage.

for the experimental group involved three sessions: 5minute premeditation relaxation (stage $\mathrm{R}$ ), 40-minute Chanmeditation practice (stage $\mathrm{M}$ ), and 5-minute Chakra focusing (stage $\mathrm{C}$ ). In stage $\mathrm{C}$, practitioners focused their mind and perception on a particular chakra named Chan Chakra (the third ventricle inside the brain, as illustrated in Appendix). No particular intervention was applied to the control group during the 50-minute EEG recording. The control subjects only sat in a relaxing position with eyes being closed, yet in the awake state.

\subsection{Signal Acquisition and Preprocessing. The EEG signals} were originally sampled at $1,000 \mathrm{~Hz}$ after being filtered by the analog, instrumentational band-pass filter with a passband of $0.5-50 \mathrm{~Hz}$. The band-pass filter setting was selected to eliminate the $60 \mathrm{~Hz}$ interference by the power lines. A high sampling rate of $1,000 \mathrm{~Hz}$ was adopted to preserve the waveform quality of gamma rhythms $(>25 \mathrm{~Hz})$ often observed in Chan-meditation EEG that had been investigated in the other study of our research group. In this study, we downsampled the EEG with a sampling rate of $200 \mathrm{~Hz}$ since the major focus of this study is the alpha-dominated EEG epochs. The segments contaminated by such artifacts as eye blinking, eyeball movement, and muscle activities were prescreened in the preprocessing stage.

Wavelet decomposition provides an effective tool to extract the particular EEG rhythm of interest [34-36]. In addition, wavelet transform (WT) possesses such appealing properties as time-frequency localization and multirate filtering. Specific EEG rhythm may be extracted by dedicatedly designing the WT parameters. Wavelet transform can be implemented either in continuous configuration (CWT) or in discrete form (DWT). Due to the problem of extremely narrow-band EEG rhythmic pattern, CWT (continuous-time wavelet transform) was implemented in our study to reliably localize the correct spectral components of alpha rhythm.

In CWT, the signal to be analyzed is matched and convolved with the continuous wavelet basis function with the continuous time and frequency. The original signal is expressed as a weighted sum of the continuous basis wavelet function digitized by the sampling rate of the corresponding scale. The basis for wavelet transform is called the mother wavelet prototype. Wavelet functions are families of functions satisfying prescribed conditions, such as continuity, zeromean amplitude, and finite or near finite duration. Some categories of wavelet functions may involve such properties like orthogonality and biorthogonality, regularity, and so forth [35-37].

Mother wavelet prototype needs to be appropriately selected according to the properties of the particular signal under investigation. Adeli et al. [38] successfully captured and localized the $3 \mathrm{~Hz}$ spike and wave complex in the epileptiform EEG by applying wavelet decomposition with Daubechies wavelets. Our previous study has corroborated the feasibility of adopting Daubechies 6 (DB6) wavelet as the mother wavelet in EEG rhythmic analysis [37].

The family of Daubechies wavelets is known for its orthogonal property and efficient implementation. The lower-order Daubechies wavelets are too coarse to properly represent EEG sharp transients. The higher-order ones with extra oscillations are beyond the requirements for analyzing the low-frequency EEG rhythms. Particularly, order 6 Daubechies wavelet becomes most appealing in our study because its waveform pattern appears to mimic the neuronal action potentials.

2.3. Nonlinear Interdependence Measure. The scheme for evaluating the nonlinear interdependence was based on the modified algorithm employed in computing the similarity index $S(\mathbf{X} \mid \mathbf{Y})$ [24]. Major tasks involved in the algorithm are reconstruction of the $m$-dimensional phase-space trajectory and computation of the average cloud radius centered at a given state point.

2.3.1. Reconstruction of $m$-Dimensional Trajectory. Consider the brain as a nonlinear dynamical system. The nonlinear interactions of the local neuronal networks can be assessed by the analysis of the collective dynamics underlying EEG time series simultaneously recorded from different brain regions. The first step is to reconstruct the multidimensional phasespace portrait of the system dynamics $\mathbf{X}$ and $\mathbf{Y}$, respectively, from EEG time series $x[i]$ and $y[i]$. According to the Takens embedding theory [39], a smooth map from the EEG time series $\{x[i] \mid i=1, \ldots, N+(m-1) \tau\}$ to the phase-space trajectory $\mathbf{X}=\left\{X_{i} \mid X_{i}=(x[i], x[i+\tau], \ldots, x[i+(m-1) \tau])\right\}_{i=1}^{N}$ preserves some important topological invariants of the original system. The reconstruction assumes a total number of $N$ system-state points in the $m$-dimensional phase-space trajectory, utilizing a rational time delay $\tau$ (in sample point) $[40,41]$. The dimension $m$ indicates the number of degrees of freedom of the nonlinear system and, accordingly, reflects the complexity of the system dynamics. 
2.3.2. Computation of the Average Cloud Radius. Consider a state point $X_{i}$ on the $m$-dimensional phase trajectory. As illustrated in Figure 2, a KNN hypersphere, formed by the $K$ 's nearest neighboring $(K N N)$ points of $\mathbf{X}_{i}$, is a cloud composed of $\mathrm{Km}$-dimensional neighboring points around $X_{i}$. Let $r_{i, j}$ and $s_{i, j}, j=1, \ldots, K$, denote the time indices of the $K N N$ points of $X_{i}$ and $Y_{i}$, respectively. Then, the set of state points in the $K N N$ hypersphere centered at $X_{i}$ is $\left\{X_{r_{i, j}} \mid j=1, \ldots, K\right\}$. The average square Euclidean distance from $X_{i}$ to its $K \mathrm{NN}$ neighbors (or the average square radius of the cloud centered at $\left.X_{i}\right)$ is defined as

$$
R_{i}^{(K)}(X)=\frac{1}{K} \sum_{j=1}^{K}\left\|X_{i}-X_{r_{i, j}}\right\|^{2},
$$

where $\|\cdot\|$ indicates the operator for calculating the Euclidean distance. Another point cloud around $X_{i}$ is formed with respect to its mutual neighbors $X_{s_{i, j}}$, which share the same temporal indexes of the KNN of $Y_{i}$. In this sense, the $\mathbf{Y}$ conditioned average square Euclidean distance is defined by replacing the true nearest neighbors of $X_{i}$ by the mutual neighbors [37]:

$$
R_{i}^{(K)}(X \mid Y)=\frac{1}{K} \sum_{j=1}^{K}\left\|X_{i}-X_{s_{i, j}}\right\|^{2} .
$$

In the extreme case of $K=N$, the average square radius of the trajectory centered at $X_{i}$ is given by

$$
R_{i}(\mathrm{X})=\frac{1}{N-1} \sum_{j=1, j \neq i}^{N}\left\|X_{i}-X_{j}\right\|^{2}
$$

Then, for two strongly synchronized systems, both self and mutual neighbors mostly coincide so that $R_{i}^{(K)}(X) \approx R_{i}^{(K)}(X \mid$ $Y) \ll R_{i}(X)$; whereas for independent systems, mutual neighbors are more scattered that leads to $R_{i}^{(K)}(\mathrm{X}) \ll R_{i}^{(K)}(\mathrm{X} \mid$ $Y) \approx R_{i}(X)$. Accordingly, the degree of interdependence of these two systems is reflected by the similarities (or dissimilarities) between these two cloud patterns formed by self and mutual neighbors. The strength of similarity between these two point clouds is termed as similarity index $S[24,37]$ and is defined as follows:

$$
S^{(K)}(X \mid Y)=\frac{1}{N} \sum_{i=1}^{N} \frac{R_{i}^{(K)}(X)}{R_{i}^{(K)}(X \mid Y)} .
$$

$S^{(K)}(X \mid Y)$ assesses the statistical dependence of the state-space structure of $\mathbf{X}$ on that of $\mathbf{Y}$ in the sense of testifying whether closeness in $\mathbf{X}$ implies closeness in $\mathbf{Y}$ and vice versa. Two identical systems with the same sets of self and mutual neighbors result in the maximum similarity index $(S=$ 1 ), whereas the index is close to zero $(S \approx 0)$ for completely independent systems. The opposite interdependence $\left(S^{(K)}(Y \mid X)\right)$ can be computed analogically. Notice that similarity indexes are in general asymmetric; that is, $S^{(K)}(Y \mid$ $X) \neq S^{(K)}(X \mid Y) . S^{(K)}(X \mid Y)$ evaluates the effect of system $\mathbf{Y}$ on system $\mathbf{X}$. From the point of view of the system theory, signal $\mathbf{Y}$ is regarded as the source or the active role in the interaction, while signal $\mathbf{X}$ plays a passive role (a sink). On the other hand, $S^{(K)}(Y \mid X)$ analysis considers $\mathbf{Y}$ as the sink that plays the passive role $[24,37]$.

The asymmetry of $S$ is one of the main advantages over the other nonlinear measures such as the mutual information and the phase synchronizations. The fact that $S$ is asymmetric allows us to study not only topographic patterns but also functional properties. By considering each EEG electrode either as a sink or as a source in the nonlinearinterdependence interaction, we may thereby further explore the brain functional topological profile and the direction of interaction among local neuronal networks [19]. For example, the condition of $S(Y \mid X)>S(X \mid Y)$ indicates that $\mathbf{Y}$ depends more on $\mathbf{X}$ than vice versa. In other words, $\mathbf{X}$ has a greater influence on $\mathbf{Y}$ than vice versa. In such a case, $\mathbf{X}$ is said to be more active and $\mathbf{Y}$ is more passive. By considering each electrode either as a sink or as a source in the nonlinear dynamical interaction, we may thereby explore the spatial direction of the interaction and the dominance of local neuronal networks under Chan meditation [42].

In order to maximize the sensitivity to the underlying synchronization and gain the robustness against noise, we proposed a modified version of $S$ measure with an adjustable range of $K N N$. Following our previous study of dimensional complexity index $[27,28]$, a reliable estimate of dimensional complexity of a system was obtained by averaging the complexity indexes over a moderate range of $K$ 's. A small $K$ causes superimposed noise, while a large $K$ results in a measurement involving multimodal effects [27]. To determine a robust measure against noise, it follows that the final estimate of nonlinear interdependence is the average $S^{(K)}(X \mid Y)$ over an appropriate range of $K$ 's and is denoted by $S(X \mid Y)$.

In the practical implementation, previous studies of dimensional complexity for meditation EEG have established a moderate choice of parameters. The time delay $\tau$ can be determined by the first zero-crossing of the corresponding autocorrelation function. Embedding dimension $m$ can be determined by the convergent estimate of dimensionality. The window length $N$ is selected to encompass the stabilization of dynamical behavior in the phase space in the sense of the convergent estimate of quantitative nonlinear dynamical property of reconstructed EEG trajectory, for example, correlation dimension. As a consequence, the implementing parameters were selected to be $\tau=5$ (sample points), $m=10$, and window length $N=1,000$ sample points ( 5 seconds) that ensure convergent and reliable estimates [24, 27, 28]. The final estimate, $S(X \mid Y)$, was obtained by averaging the $S^{(K)}(X \mid Y)$ for $K$ ranging from 20 to 35 .

2.3.3. Outline of the Scheme. The entire scheme employed in this study is illustrated in Figure 3 that integrates different theories and methods to evaluate the nonlinear interdependence for multichannel EEG.

To investigate the nonlinear-interdependent behaviors of alpha activities, CWT is employed to identify alphadominated epochs in the entire EEG record. An EEG segment 


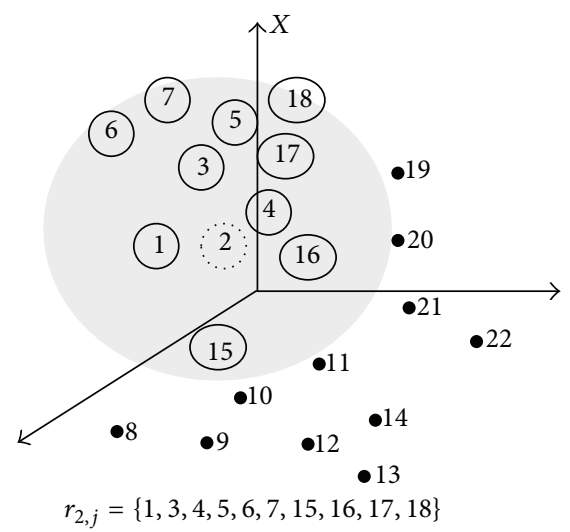

(a)

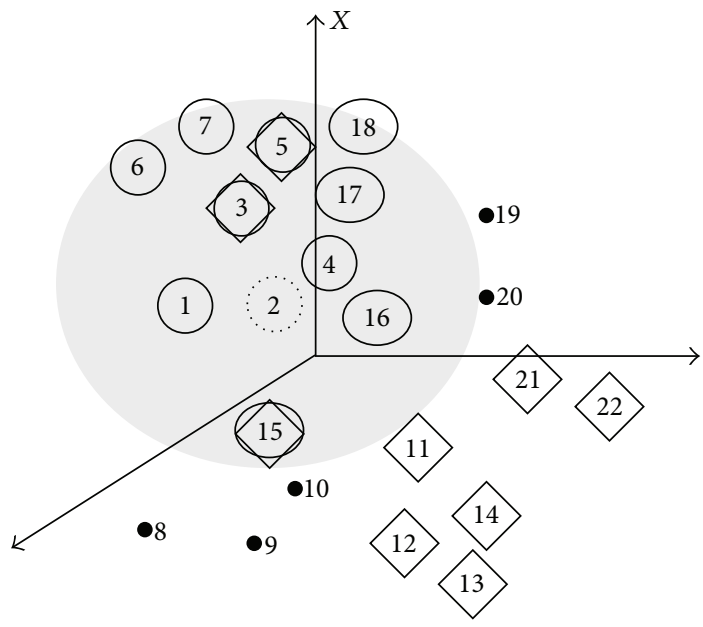

(c)

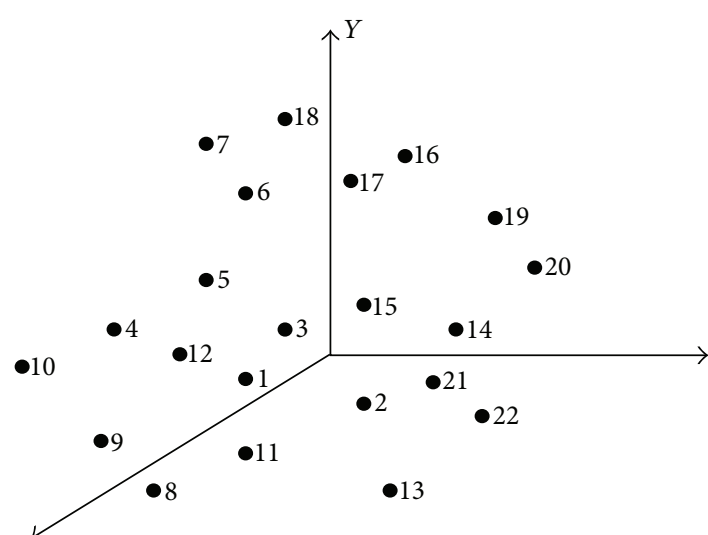

(b)

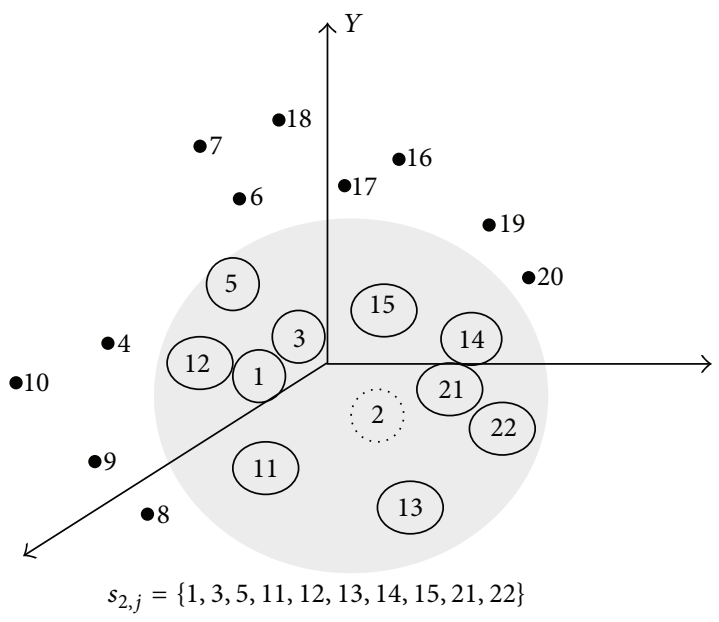

(d)

FIgure 2: Illustration for (a) self neighbors $X_{r_{2, j}}(\bigcirc)$, (b) state points in $Y$, and (c) mutual neighbors $X_{s_{2, j}}(\diamond)$ where the indexes $s_{2, j}$ are determined from the indexes of (d) $K N N$ of $Y_{2}(K=10)$, assuming $m=3, K=10, i=2$, and $N=22$.

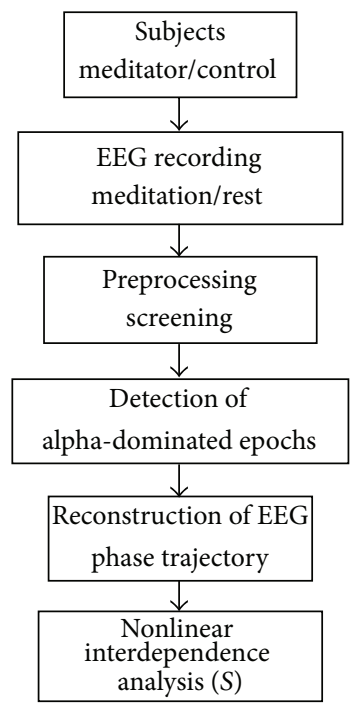

FIGURE 3: Scheme for evaluating nonlinear interdependence of multichannel EEG. is identified to be alpha dominant if the percentage of $\alpha$ power to the total power is at least $50 \%$ in more than 15 channels (one half of the total channels). Figure 4 displays the results of interpreting the 5-second EEG recorded from channels $\mathrm{Oz}$, $\mathrm{Cz}$, and Fz. The alpha-power percentage (denoted as $\rho$ ) for each one-second epoch is listed beneath the EEG tracing. The 5-second EEG tracing is plotted with the amplitude ranging from $-50 \mu \mathrm{V}$ to $50 \mu \mathrm{V}$. Parameter $\rho$ evaluated for different channels may reflect the focalized behavior of alpha activity.

To extend the capacity of assessing the neural-network interaction, the source $X_{j}$ can be generalized as an integrated local network involving $L$ active electrode sites so that $S_{p}\left(X_{i}\right)$ becomes the average of $L$ 's $S\left(X_{i} \mid X_{j}\right)$, assuming $X_{j} \neq X_{i}$ :

$$
S_{p}\left(X_{i}\right)=\frac{1}{L} \sum_{j} S\left(X_{i} \mid X_{j}\right) .
$$

Equation (5) then evaluates the integrative effects of $L$ active electrodes on $X_{i}$. On the other hand, the influence of an active 


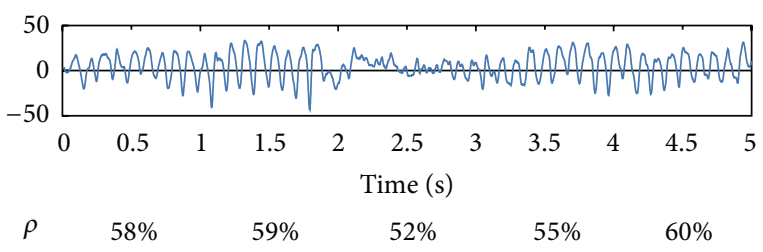

(a) $\mathrm{Oz}$

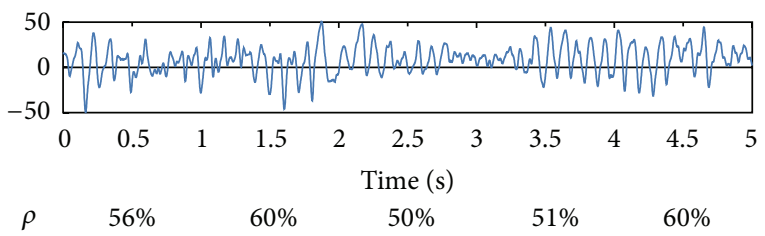

(b) $\mathrm{Cz}$

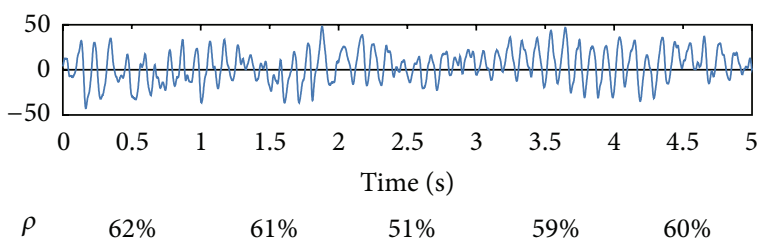

(c) $\mathrm{Fz}$

FIGURE 4: Percentage of alpha power to total power for each onesecond epoch of the five-second EEG segments (amplitude in $\mu \mathrm{V}$ ) recorded from (a) $\mathrm{Oz}$, (b) $\mathrm{Cz}$, and (c) Fz.

electrode $X_{j}$ on the integrative neural network encompassed by $L$ passive electrodes $\left(X_{i}\right)$ can be evaluated by

$$
S_{a}\left(X_{j}\right)=\frac{1}{L} \sum_{i} S\left(X_{i} \mid X_{j}\right)
$$

assuming $X_{i} \neq X_{j}$. Both $S_{p}\left(X_{i}\right)$ and $S_{a}\left(X_{j}\right)$ are called regional interdependence index $(R I I)$.

In Chan-meditation practice, practitioners often focus on five regions alternately, frontal, posterior, left, right, and central regions, after activating the Chan Chakra inside the third ventricle. The purpose is to eliminate the stream of jumbled thoughts and produce a tranquil mind. To investigate the effect of such regional focusing, we accordingly divided 30 EEG recording sites into five regions:

$$
\begin{aligned}
& \text { frontal (F): Fp1, Fp2, F7, F3, Fz, F4, and F8; } \\
& \text { posterior (P, Parietal + Occipital): O1, Oz, O2, P7, P3, } \\
& \text { Pz, P4, and P8; } \\
& \text { central (C): FCz, Cz, and CPz; } \\
& \text { left Temporal (LT): FC3, FT7, T7, C3, TP7, and CP3; } \\
& \text { right Temporal (RT): FC4, FT8, T8, C4, TP8, and CP4. }
\end{aligned}
$$

\section{Results and Discussion}

3.1. Interdependence Matrix of Chan-Meditation EEG. Consider a given source signal $\mathrm{Y}$. The influence of source signal $\mathbf{Y}$ on sink signal $\mathbf{X}, S(\mathbf{X} \mid \mathbf{Y})$, can be expressed as a $30 \times$ 30 interdependence matrix with the element $S_{i j}=S\left(X_{i} \mid Y_{j}\right)$ denoting the coupling strength of interaction of the source $Y_{j}$ affecting the sink $X_{i}$. The similarity index (S.I.) was calculated for $870(30 \times 29)$ electrode pairs. As displayed in Figure 5(a), the color image encoded the quantities in the 30 $\times 30$ interdependence matrix $\mathbf{S}$. The right-side color chart encodes the strength level of $S_{i j}$, from blue to red indicating the range of $S$ from the smallest to the largest value. EEG channels are in the order of (from top/left): O2, Oz, O1, P7, P3, Pz, P4, P8, TP8, CP4, CPz, CP3, TP7, T7, C3, Cz, C4, T8, FT8, FC4, FCz, FC3, FT7, F7, F3, Fz, F4, F8, Fp2, and Fp1. For example, the box at the lower-left corner characterizes the effect of $\mathrm{O} 2$ channel on Fp1 channel, as denoted by S(Fp1 I $\mathrm{O} 2$ ). Accordingly, the first row reveals the effect of source $\mathrm{O} 2$, $\mathrm{Oz}, \ldots$, and Fp1, respectively, on sink O2. On the other hand, the first column indicates how source $\mathrm{O} 2$ affects sink $\mathrm{O} 2, \mathrm{Oz}$, ..., and Fp1, respectively. The dark red along the diagonal line indicates the highest similarity index $S=1$ when the source and sink signals are identical.

This figure exhibits some typical behavior in the $S$ matrix; that is, stronger interdependence occurs in the pairs of nearby EEG channels. On the other hand, weaker interaction is measured as two channels are much apart. Moreover, box $(i, j)$ does not equal its transposed partner box $(j, i)$, indicating the asymmetry of $S$ matrix. Figures $5(\mathrm{~b})-5(\mathrm{~d})$ display the top view of brain topographic mapping of $S_{a}(\mathrm{FP} 1)$, $S_{a}(\mathrm{FP} 2)$, and $S_{a}(\mathrm{Oz})$ extracted, respectively, from the 30th, 29th, and 2nd columns of Figure 5(a). The topographic mapping was plotted by the function topoplot.m provided by EEGLab. The mappings exhibit the efficacy of the given channel acting as the source role. The results in Figures 5(b) to 5(d) reveal the right-frontal dominance. The occipital channels are comparably less active with respect to the frontal neuronal networks. Such weaker influence of occipital and posterior regions on the other regions can be clearly observed from the blue color dominating in the left three columns of $\mathbf{S}$ matrix (Figure 5(a)), corresponding to the source at $\mathrm{O} 2, \mathrm{Oz}$, and $\mathrm{O} 1$.

3.2. Inter-Region Interdependence Analysis-Experimental Group. Inter-regional nonlinear interdependence was analyzed for EEG recorded in three different sessions (stage R, $\mathrm{M}$, and $\mathrm{C}$ ). Due to the premeditation brain-drilling practice described in previous section, we particularly focused on the left-right temporal (LT-RT) and frontal-posterior (F-P) neural-network interactions. For example, $S(\mathrm{~F} \rightarrow \mathrm{P})$ is computed by averaging all $S_{a}\left(X_{j}\right)$ in (6) for all $X_{j} \in \mathrm{F}$ and $X_{i} \in \mathrm{P}$ to assess the integrative source effect of all electrodes in frontal region driving the posterior region. On the other hand, $S(\mathrm{P} \rightarrow \mathrm{F})$ is computed by averaging all $S_{a}\left(X_{j}\right)$ in (6) for all $X_{j} \in \mathrm{P}$ and $X_{i} \in \mathrm{F}$ when all the electrodes in the posterior region play the source role to drive the frontal region.

Table 1 lists the group averages and standard deviations of $S(\mathrm{~F} \rightarrow \mathrm{P}), S(\mathrm{P} \rightarrow \mathrm{F}), S(\mathrm{LT} \rightarrow \mathrm{RT})$, and $S(\mathrm{RT} \rightarrow$ LT) at three experimental stages (R, M, and C). Evidently, $S(\mathrm{~F} \rightarrow \mathrm{P})$ is consistently greater than $S(\mathrm{P} \rightarrow \mathrm{F})$ for all three stages. The $P$ values $(0.0055,0.0031$, and 0.0302$)$ of paired sample $t$-test are all smaller than 0.05 , that demonstrates the 


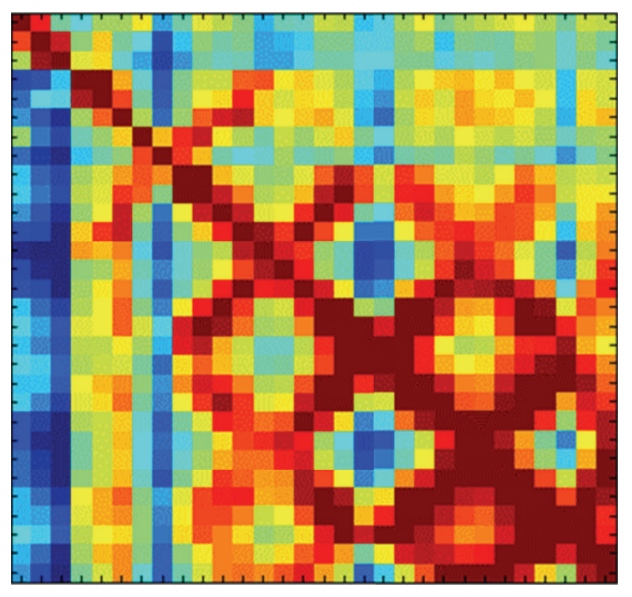

(a)

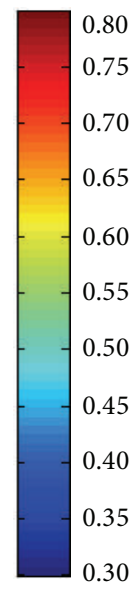

0.30

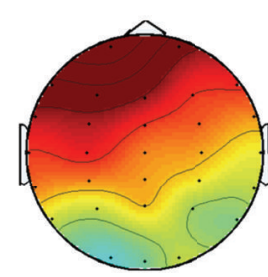

(b) $S_{a}$ (FP1)

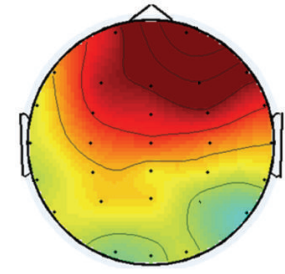

(c) $S_{a}(\mathrm{FP} 2)$

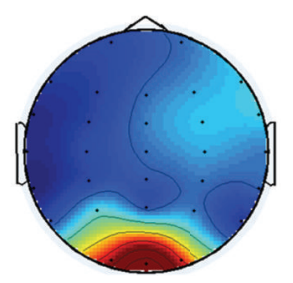

(d) $S_{a}(\mathrm{Oz})$

FIGURE 5: S.I. analysis for experienced practitioner during Chan meditation (Stage M) (a) $30 \times 30 \mathrm{~S}$ matrix, and brain topographical mappings (top view) of (b) $S_{a}$ (FP1), (c) $S_{a}(\mathrm{FP} 2)$, and (d) $S_{a}(\mathrm{Oz})$, indicating the average driving strength of the EEG sites FP1, FP2, and Oz.

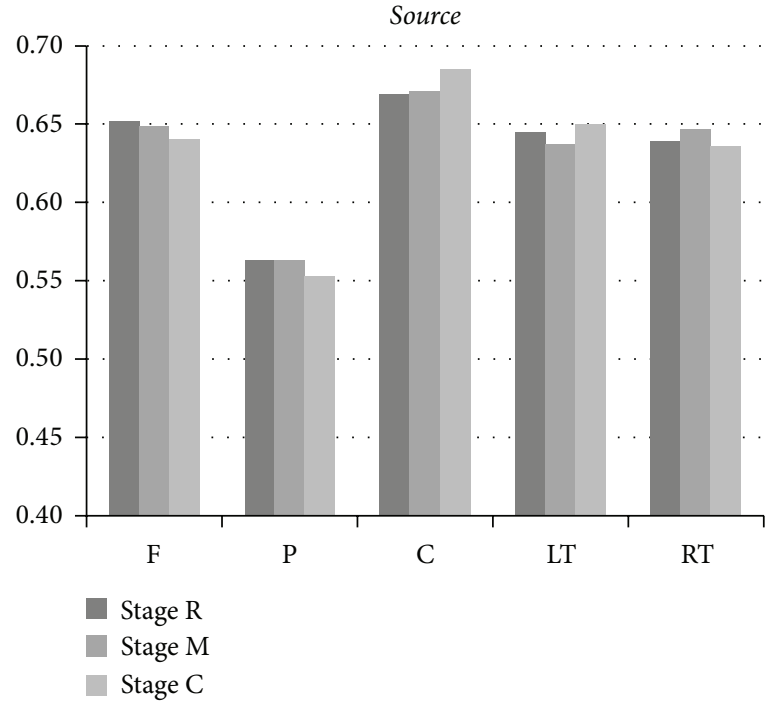

(a)

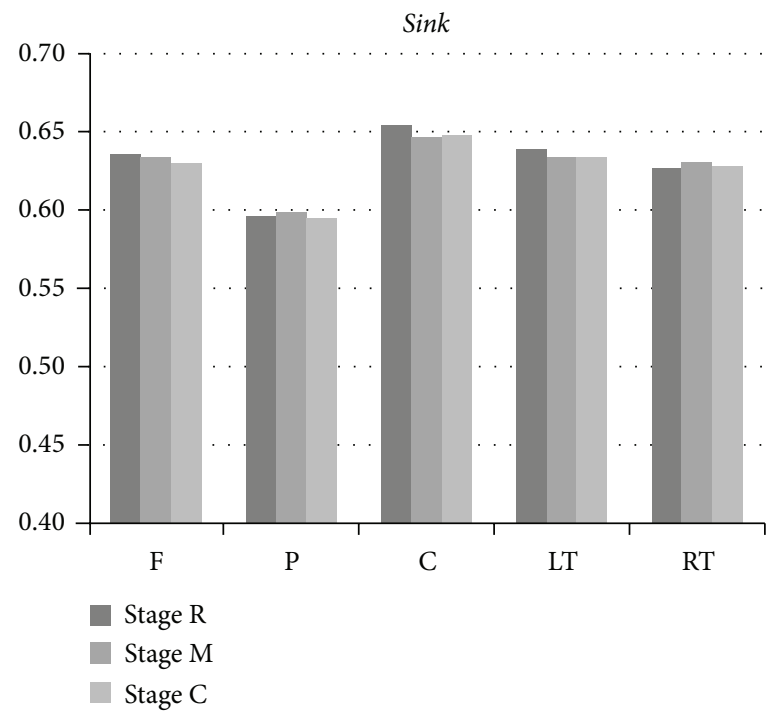

(b)

FIGURE 6: Average effectiveness of each region playing the role of a source (a) and sink (b). Three bars in each RII cluster correspond to three experimental stages.

statistical significance of frontal-alpha dominance at all three stages. On the other hand, results of the left-right temporal analysis of nonlinear interdependence reveal no distinctly dominant role of the laterally neural-network operation, that is, $S(\mathrm{LT} \rightarrow \mathrm{RT}) \approx S(\mathrm{RT} \rightarrow \mathrm{LT})$. The higher $P$ values for the $S(\mathrm{LT} \rightarrow \mathrm{RT})-S(\mathrm{RT} \rightarrow \mathrm{LT})$ paired $t$-test indicate no statistically significant difference between two sets of results. We may further infer the balancing operations between the left-brain and right-brain hemispheres.

Our results demonstrate that interactions between left and right hemispheres are much more intensive than the interactions between frontal and posterior regions, with $P=$ 0.0016 , considering all the experimental subjects at all three stages.
Figure 6 provides an alternative viewpoint for exploring how a given region of interest ROI (F, P, C, LT or RT) influences or is influenced by the other regions. In Figure 6, left (right) group of five 3-bar clusters corresponds to the average effectiveness of each region playing the active (passive) role at three stages. For example, the leftmost bar indicates the average of $S(\mathrm{~F} \rightarrow \mathrm{P}), S(\mathrm{~F} \rightarrow \mathrm{LT}), S(\mathrm{~F} \rightarrow$ $\mathrm{RT})$, and $S(\mathrm{~F} \rightarrow \mathrm{C})$ for stage $\mathrm{R}$, while the rightmost bar indicates the average of $S(\mathrm{~F} \rightarrow \mathrm{RT}), S(\mathrm{P} \rightarrow \mathrm{RT})$, $S(\mathrm{C} \rightarrow \mathrm{RT})$, and $\mathrm{S}(\mathrm{LT} \rightarrow \mathrm{RT})$ for stage C. Among all five regions, posterior region, as either the source or sink, apparently exhibits the weakest link to the other regions. In addition, the effectiveness of active role of posterior region is weaker than that of passive role. The results strongly 
TABLE 1: Group averages and standard deviations of $S(\mathrm{~F} \rightarrow \mathrm{P}), S(\mathrm{P} \rightarrow \mathrm{F}), S(\mathrm{LT} \rightarrow \mathrm{RT})$, and $S(\mathrm{RT} \rightarrow \mathrm{LT})$ at three experimental stages $(\mathrm{R}, \mathrm{M}$, and $\mathrm{C})$, including the $P$ values of student $t$-test for $S(\mathrm{~F} \rightarrow \mathrm{P})-S(\mathrm{P} \rightarrow \mathrm{F})$ and $S(\mathrm{LT} \rightarrow \mathrm{RT})-S(\mathrm{RT} \rightarrow \mathrm{LT})$ pairs.

\begin{tabular}{|c|c|c|c|c|}
\hline Stage & $S(\mathrm{~F} \rightarrow \mathrm{P})$ & $S(\mathrm{P} \rightarrow \mathrm{F})$ & $S(\mathrm{LT} \rightarrow \mathrm{RT})$ & $S(\mathrm{RT} \rightarrow \mathrm{LT})$ \\
\hline \multicolumn{5}{|c|}{$\bar{R}$} \\
\hline Group average & 0.56 & 0.51 & 0.60 & 0.61 \\
\hline Group std & 0.05 & 0.05 & 0.07 & 0.05 \\
\hline$P$ value & \multicolumn{2}{|c|}{0.0055} & \multicolumn{2}{|c|}{0.3476} \\
\hline \multicolumn{5}{|l|}{$\mathrm{M}$} \\
\hline Group average & 0.56 & 0.50 & 0.60 & 0.61 \\
\hline Group std & 0.03 & 0.06 & 0.06 & 0.04 \\
\hline$P$ value & \multicolumn{2}{|c|}{0.0031} & \multicolumn{2}{|c|}{0.1987} \\
\hline \multicolumn{5}{|c|}{$\mathrm{C}$} \\
\hline Group average & 0.55 & 0.49 & 0.61 & 0.61 \\
\hline Group std & 0.07 & 0.06 & 0.06 & 0.06 \\
\hline$P$ value & \multicolumn{2}{|c|}{0.0302} & \multicolumn{2}{|c|}{0.3953} \\
\hline
\end{tabular}

suggest the inactive behaviors of parietal-occipital lobes since region $\mathrm{P}$ encompasses the EEG-electrode sites of parietal and occipital lobes. The parietal lobe is responsible for integrating sensory information from various parts of the body, with the particular functions of determining spatial sense and navigation. Functions of occipital lobe mainly include visual reception, visual-spatial processing, and color recognition. As described previously, the core essence of orthodox Chanmeditation practice is to transcend physiological, mental, and all states of consciousness to prove the existence of true being. The inactive posterior regions may provide the evidence of brain rewiring in preparation for such transcendence.

Region C encompasses three midline electrodes locating from precentral to postcentral cortex. Region C, as the source, apparently dominates over the other four regions regardless of the stages. On the other hand, region $\mathrm{C}$ as the passive role is affected mostly among the five regions. Region $\mathrm{C}$ constantly exhibits the largest $R I I$ at all stages.

We may draw a tentative hypothesis from the mechanism of Chan-meditation practice. Practitioners are required to keep Chan Chakra active at any moment, that results in the formation of an energy pathway between Chan Chakra and Qian-Ding acupoint on scalp (Figure 10(b)). Does such physiological reformation correlate to the significant effectiveness of region $\mathrm{C}$ ? It leaves an open question for future investigation.

RII characterizing the regional interdependence behaves differently for each region when the experimental subjects switch their mental states from $\mathrm{R}$ (resting) to $\mathrm{M}$ (meditation) or from $\mathrm{R}$ to $\mathrm{C}$ (Chakra focusing). To investigate the effect of different experimental sessions, the RII percentage increase/decrease from stage $\mathrm{R}$ to $\mathrm{M}$ and from stage $\mathrm{R}$ to $\mathrm{C}$ were computed for each of the five regions (F, P, C, LT, and RT) acting as either the active or the passive role. In comparison of RII between stage $\mathrm{M}$ and stage $\mathrm{R}$, the percentage larger than $1 \%$ was observed in the regions of $\operatorname{LT}_{\text {active }}(-1.24 \%)$, $\mathrm{RT}_{\text {active }}(1.25 \%)$, and $\mathrm{C}_{\text {passive }}(-1.07 \%)$. On the other hand, the regions of significant change in $R I I$, when comparing stage $\mathrm{C}$ with $\mathrm{R}$, include $\mathrm{F}_{\text {active }}(-1.84 \%), \mathrm{P}_{\text {active }}(-1.78 \%)$, and $\mathrm{C}_{\text {active }}(2.39 \%)$. On the basis of RII of stage $\mathrm{R}$ for each individual region, we summarize the changes of $R I I$ at stages $\mathrm{M}$ and $\mathrm{C}$ as follows.

(1) In the active-role analysis, region LT becomes more deactivated at stage $\mathrm{M}$, while region RT becomes more activated. When meditation subjects focused on Chan Chakra, the active driving strength of region $\mathrm{C}$ increases significantly (2.39\%). On the other hand, suppression of the source activity occurs to both regions $\mathrm{F}$ and $\mathrm{P}$ (the regions anterior to and posterior to region $\mathrm{C}$ ).

(2) In the passive-role analysis, only region $\mathrm{C}$ becomes notably deactivated at stage $M$ (free meditation). In general, differences are trivial in comparison of $R I I_{\text {passive }}$ between stages $\mathrm{M}$ and $\mathrm{R}$.

(3) Chan meditation deactivates the left brain hemisphere, whereas it inactivates the right brain hemisphere.

Except for region $\mathrm{P}$, the active-role effectiveness of a given region is better than its passive-role effectiveness.

3.3. Inter-Region Interdependence Analysis-Control Group. In control group, the group average and standard deviation of $S(\mathrm{~F} \rightarrow \mathrm{P}), S(\mathrm{P} \rightarrow \mathrm{F}), S(\mathrm{LT} \rightarrow \mathrm{RT})$, and $S(\mathrm{RT} \rightarrow \mathrm{LT})$ at stage $R$ are, respectively, $0.55 \pm 0.04,0.51 \pm 0.04,0.59 \pm 0.03$, and $0.58 \pm 0.04$. The $P$ values of student $t$-test for $S(\mathrm{~F} \rightarrow$ $\mathrm{P})-S(\mathrm{P} \rightarrow \mathrm{F})$ and $S(\mathrm{LT} \rightarrow \mathrm{RT})-S(\mathrm{RT} \rightarrow \mathrm{LT})$ pairs are 0.002 and 0.457 . The results also reveal the frontal dominance and left-right lateral balance for the control group at rest. Yet, compared with the Chan-meditation practitioners, control group exhibits weaker strength of effectiveness no matter if the region plays an active or a passive role.

To explore the average effectiveness of a given ROI, we averaged the RIIs for the region in connection with the other four regions. Figure 7 displays how a given ROI (F, P, C, LT, or RT) influences (active) or is influenced (passive) by the other regions. Similar to the results of experimental group, region $\mathrm{P}$ as either the active or passive role exhibits the weakest links to the other regions. 


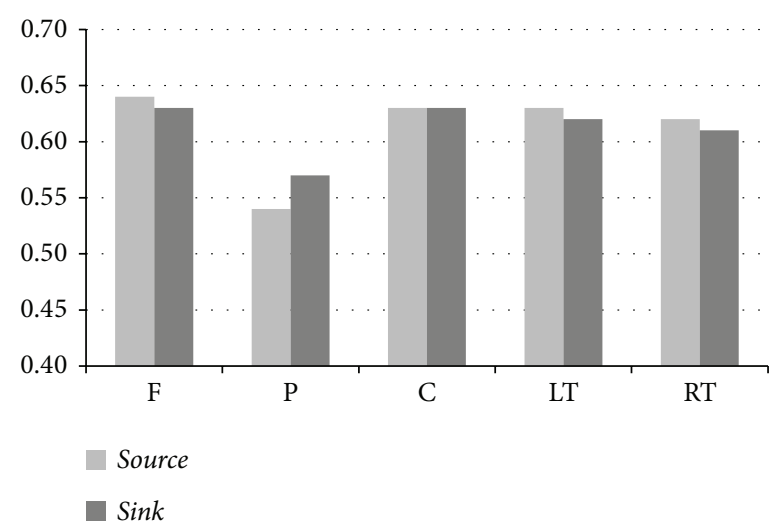

FIGURE 7: Average effectiveness of each region playing either the active or passive role.

Comparing the efficacy of two counteractive roles played by the same region, we observe that the source-role effectiveness of a given ROI is higher than its sink-role effectiveness except region $\mathrm{P}$.

\subsection{Comparison between Experimental and Control Groups.}

Figure 8 illustrates the group averages of RIIs, including $S(\mathrm{~F} \rightarrow \mathrm{P}), S(\mathrm{P} \rightarrow \mathrm{F}), S(\mathrm{LT} \rightarrow \mathrm{RT})$, and $S(\mathrm{RT} \rightarrow \mathrm{LT})$, for the experimental group at three stages $(R, M$, and $C)$ and for the control group at rest. Experimental group reveals much more intensive lateral (LT $\longleftrightarrow$ RT) interactions than control group. The differences are statistically significant for experimental group at stage $\mathrm{M}(P=0.0336)$ and at stage $\mathrm{C}$ $(P=0.0411)$. On the other hand, region $\mathrm{P}$ responsible for spatial sense and navigation becomes comparatively inactive for Chan-meditation practitioners at stage $\mathrm{C}$.

The assembling illustration in Figure 9 is used to compare the average effectiveness of each region between two groups. Experimental group, playing either a source role or a sink role, apparently exhibits higher average effectiveness in all five regions. The extraordinarily large RIIs for region C, particularly acting the source role, may be assumed to be correlated with the strengthening of neural networks of region $\mathrm{C}$ dominating over the other regions through the spiritual focusing on Chan Chakra. According to the postexperimental interview with Chan-meditation practitioners, such central $(\mathrm{FCz}-\mathrm{Cz}-\mathrm{CPz})$ dominating behavior could be linked to the Chan-Chakra activation that further induces the perception of grand, solemn energy flow in and out through the cortical regions defined by acupoints DU20 (Baihui), DU21 (Qianding), and DU22 (Xinhui) in TCM (traditional Chinese Medicine). Figure 10(b) (Appendix) illustrates the locations of these three acupoints.

\section{Conclusion}

The time-transcending, nonmaterial sacred spiritual experiences of Chan-meditation practitioners bring our attention to the study of the unique interactions among regional neural networks in the brain. Scientific approach to the scope of

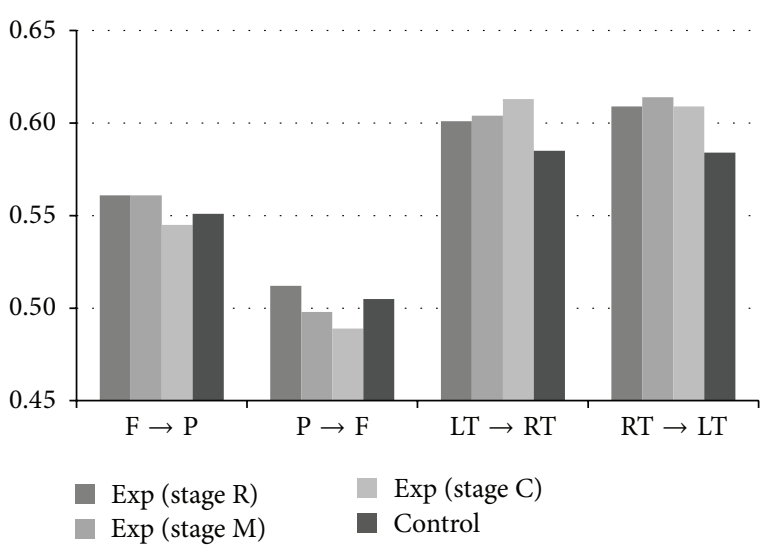

Figure 8: Group averages of RIIs $(S(\mathrm{~F} \rightarrow \mathrm{P}), S(\mathrm{P} \rightarrow \mathrm{F}), S(\mathrm{LT} \rightarrow$ $\mathrm{RT})$, and $S(\mathrm{RT} \rightarrow \mathrm{LT})$ ) for experimental group at three stages and for control group at rest.

Chan meditation provides insight into the mechanism in addition to the vague sketch of meditation sensation and its multiform benefits to human beings. This paper presents our preliminary results, based on nonlinear dynamical theory, of exploring the spatial interactions among brain local neural networks under alpha-rhythmic oscillation. Quantification of nonlinear interdependence based on similarity index reveals significant intergroup difference. Significant higher lateral interactions between left and right temporal regions were observed in Chan-meditation practitioners at the stages of Chan meditation and Chakra-focusing practice. In Chan practice, practitioners follow the doctrine that the mind can be enlightened only if it surrenders its leadership power to the "heart" (Bodhi, the true self with eternal wisdom). They accordingly can experience better balance and integration of the brain hemispheres through years of Chan-meditation practice.

Chan-Chakra spiritual focusing (at stage C) remarkably strengthens the central neural-network dominance over the other regions. On the other hand, suppression of the source activity in regions $\mathrm{F}$ and $\mathrm{P}$ at stage $\mathrm{C}$ appears to reveal the meditation state of transcending the realm of physical body and mind. The particular central $(\mathrm{FCz}-\mathrm{Cz}-\mathrm{CPz})$ dominating phenomenon is reflected in long-term Chan practitioners as one of the metamorphosing processes that opens the energy pathway between Chan Chakra and the central-line scalp from acupoint DU20 to DU22 (Figure 10(b) in Appendix). In the case, practitioners experience tranquil brain and calm mind in every moment. Chan-meditation practice is to realize a Chan-style brain and Chan-style physical body, instead of merely sitting still for one hour to pursue temporary peace of mind and relief of body.

\section{Appendix}

Chan meditation originating more than 2,500 years ago has been proved to benefit the health while on the way toward the ultimate Buddhahood state. Buddha Shakyamuni disclosed the eternal truth, the supreme wisdom, the noumenal energy, 


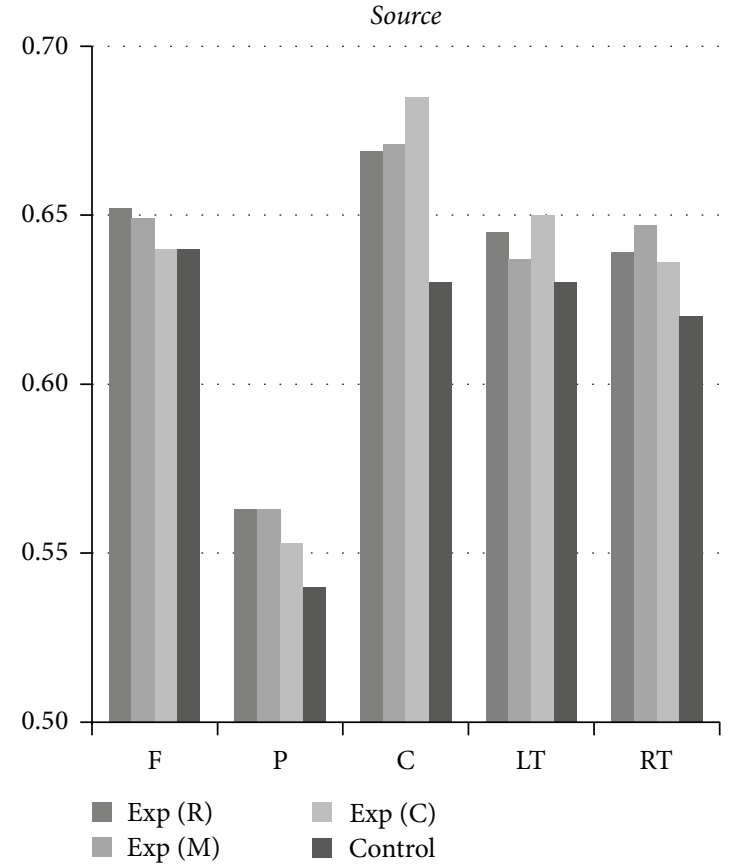

(a)

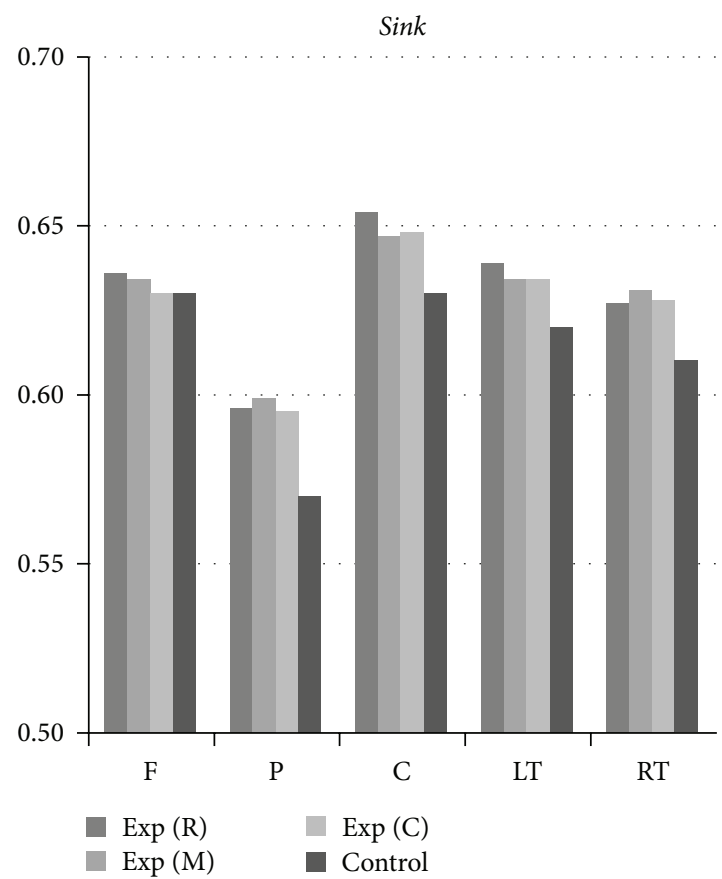

(b)

FIGURE 9: Comparison of average effectiveness of each region, as a source (a) or a sink (b), between experimental and control groups.

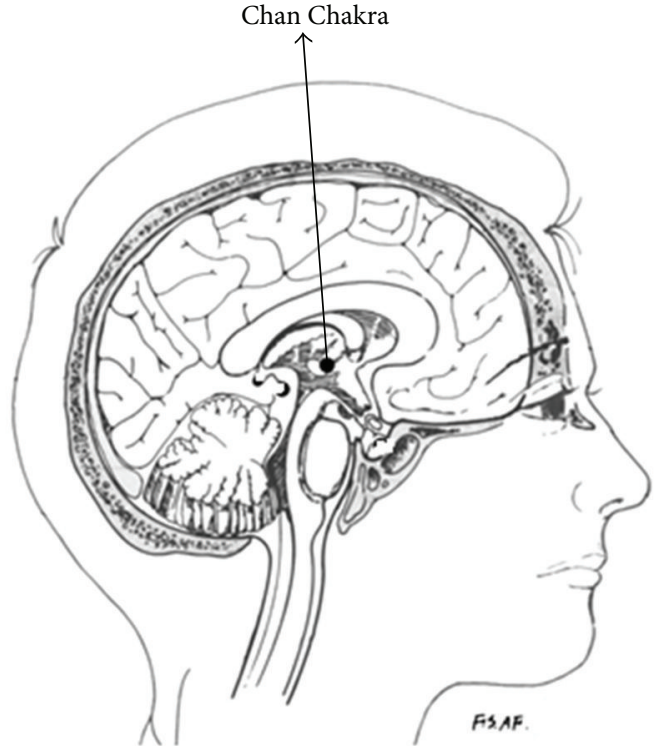

(a)

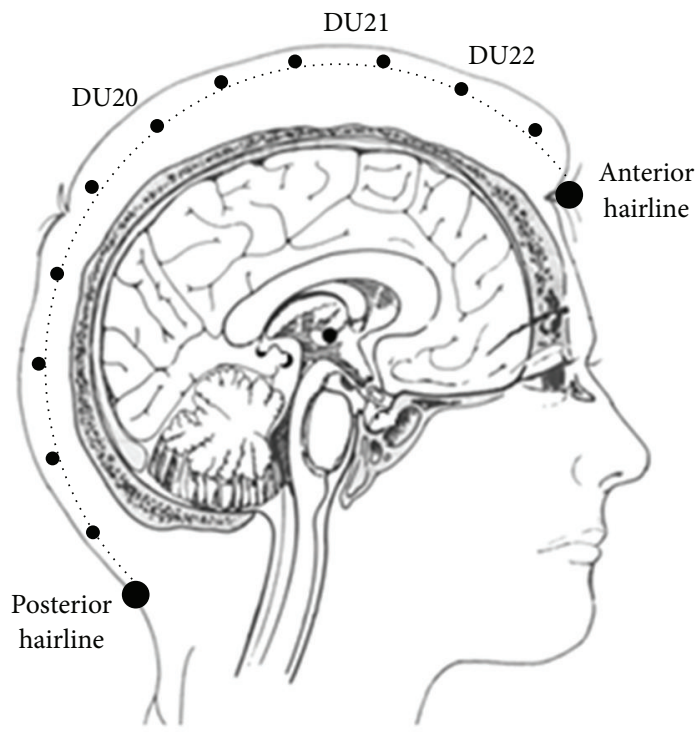

(b)

FIGURE 10: (a) Location of the Chan Chakra (inside the third ventricle). (b) Illustration of acupoints DU20 (Baihui), DU21 (Qianding), and DU22 (Xinhui) on DU meridian.

and the natural powers of the universe in Chan meditation under a linden tree. The orthodox Chan Buddhism was originated by such an exceptional affair that Buddha Shakyamuni transmitted this light of supreme wisdom to the Great Kashiyapa. The same path towards perfect enlightenment (Buddhahood) was promulgated in mainland China in 527 by Bodhidharma, the 28th patriarch. The current patriarch is Chan master Wu Jue Miao Tian, the 85th patriarch of the orthodox Chan-Buddhism Sect since the Great Kashiyapa. In orthodox Chan-Buddhist practice, very few disciples were able to catch the quintessence since it cannot be taught in any form of lectures. Written material and spoken words 
cannot promulgate the true wisdom of Chan, which can only be conveyed by the Buddhist Heart-seal Imprint from a true master.

In Chan meditation, practitioners aim to attain the true self (Buddha nature) with eternal wisdom (Bodhi) through body-mind-soul purification. Substantially speaking, such purification procedure involves the journal of transcending the physiological state (five sensory organs), the mental activities and normal consciousness, the subliminal (the manas) consciousness, and the Alaya state at which practitioners are able to perceive the sacred light emitted from Buddha nature. Buddhist Heart-seal Imprint from the Chan Patriarch is a must to assist in the purification and accomplishment. To prepare for attaining such realm, practitioners meditate with full-lotus, half-lotus, or leg-crossing posture and sit still to cultivate spiritual Reiki for penetrating into the ten important Chakras. In the course of Chan meditation, practitioners must switch their normal, chest breathing to the NavelChakra breathing (also called "fetal breathing") that is the breathing scheme for entering into deep meditation. Among the ten Chakras, Chan Chakra locating inside the third ventricle is the Buddhist paradise implemented in our body. Figure 10(a) illustrates the location of Chan Chakra.

The cun-measurand system is normally used to measure and locate the acupoints. To determine the locations of acupoints DU20, DU21, and DU22 on Governor Vessel meridian (DU meridian), we first measure the scalp-midline length between anterior hairline and posterior hairline, that is divided into 12 cuns. The locations are defined as follows:

DU20: 7 cuns above the posterior hairline and 5 cuns above the anterior hairline;

DU21: 3.5 cuns directly above the anterior hairline or 1.5 cuns anterior to DU20;

DU22: 2 cuns posterior to the anterior hairline or 3 cuns anterior to DU20.

\section{Acknowledgments}

The authors would like to thank Shung-Yu Yo for his assistance in data analysis. Chan-meditation practitioners of the Shakyamuni Buddhist Foundation are gratefully acknowledged for their enthusiastic participation in this research as volunteers. This research was supported by the grants from the National Science Council of Taiwan (Grant no.: NSC 1002221-E-009-006-MY2).

\section{References}

[1] T. Yu, H. L. Tsai, and M. L. Hwang, "Suppressing tumor progression of in vitro prostate cancer cells by emitted psychosomatic power through Zen meditation," American Journal of Chinese Medicine, vol. 31, no. 3, pp. 499-507, 2003.

[2] K. H. Coker, "Meditation and prostate cancer: integrating a mind/body intervention with traditional therapies," Seminars in Urologic Oncology, vol. 17, no. 2, pp. 111-118, 1999.

[3] D. Lester, "Zen and happiness," Psychological Reports, vol. 84, no. 2, pp. 650-651, 1999.
[4] C. R. K. MacLean, K. G. Walton, S. R. Wenneberg et al., "Effects of the transcendental meditation program on adaptive mechanisms: changes in hormone levels and responses to stress after 4 months of practice," Psychoneuroendocrinology, vol. 22, no. 4, pp. 277-295, 1997.

[5] G. A. Tooley, S. M. Armstrong, T. R. Norman, and A. Sali, "Acute increases in night-time plasma melatonin levels following a period of meditation," Biological Psychology, vol. 53, no. 1, pp. 69-78, 2000.

[6] Y.-Y. Tang, Y. Ma, Y. Fan et al., "Central and autonomic nervous system interaction is altered by short-term meditation," Proceedings of the National Academy of Sciences of the United States of America, vol. 106, no. 22, pp. 8865-8870, 2009.

[7] A. Lutz, H. A. Slagter, N. B. Rawlings, A. D. Francis, L. L. Greischar, and R. J. Davidson, "Mental training enhances attentional stability: neural and behavioral evidence," Journal of Neuroscience, vol. 29, no. 42, pp. 13418-13427, 2009.

[8] S. M. Bertisch, C. C. Wee, R. S. Phillips, and E. P. McCarthy, "Alternative mind-body therapies used by adults with medical conditions," Journal of Psychosomatic Research, vol. 66, no. 6, pp. 511-519, 2009.

[9] W. R. Marchand, "Mindfulness-based stress reduction, mindfulness-based cognitive therapy, and zen meditation for depression, anxiety, pain, and psychological distress," Journal of Psychiatric Practice, vol. 18, no. 4, pp. 233-2252, 2012.

[10] B. R. Cahn and J. Polich, "Meditation states and traits: EEG, ERP, and neuroimaging studies," Psychological Bulletin, vol. 132, no. 2, pp. 180-211, 2006.

[11] F. Travis and J. Shear, "Focused attention, open monitoring and automatic self-transcending: categories to organize meditations from Vedic, Buddhist and Chinese traditions," Consciousness and Cognition, vol. 19, no. 4, pp. 1110-1118, 2010.

[12] P.-C. Lo, M.-L. Huang, and K.-M. Chang, "EEG alpha blocking correlated with perception of inner light during Zen meditation," American Journal of Chinese Medicine, vol. 31, no. 4, pp. 629-642, 2003.

[13] H. C. Liao and P. C. Lo, "Investigation on spatiotemporal characteristics of zen-meditation EEG rhythms," Journal of International Society of Life Information Science, vol. 25, no. 1, pp. 63-71, 2007.

[14] H.-Y. Huang and P.-C. Lo, "EEG dynamics of experienced zen meditation practitioners probed by complexity index and spectral measure," Journal of Medical Engineering and Technology, vol. 33, no. 4, pp. 314-321, 2009.

[15] E. K. St. Louis and E. P. Lansky, "Meditation and epilepsy: a still hung jury," Medical Hypotheses, vol. 67, no. 2, pp. 247-250, 2006.

[16] L. I. Aftanas and S. A. Golocheikine, "Human anterior and frontal midline theta and lower alpha reflect emotionally positive state and internalized attention: high-resolution EEG investigation of meditation," Neuroscience Letters, vol. 310, no. 1, pp. 57-60, 2001.

[17] K. Ansari-Asl, J.-J. Bellanger, F. Bartolomei, F. Wendung, and L. Senhadji, "Time-frequency characterization of interdependencies in nonstationary signals: application to epileptic EEG," IEEE Transactions on Biomedical Engineering, vol. 52, no. 7, pp. 1218-1226, 2005

[18] F. Gans, A. Y. Schumann, J. W. Kantelhardt, T. Penzel, and I. Fietze, "Cross-modulated amplitudes and frequencies characterize interacting components in complex systems," Physical Review Letters, vol. 102, no. 9, Article ID 098701, 2009. 
[19] J. Bhattacharya, H. Petsche, and E. Pereda, "Interdependencies in the spontaneous EEG while listening to music," International Journal of Psychophysiology, vol. 42, no. 3, pp. 287-301, 2001.

[20] C. J. Stam, "Nonlinear dynamical analysis of EEG and MEG: review of an emerging field," Clinical Neurophysiology, vol. 116, no. 10, pp. 2266-2301, 2005.

[21] W. Singer, "Consciousness and the binding problem," Annals of the New York Academy of Sciences, vol. 929, pp. 123-146, 2001.

[22] D. Calitoiu, B. J. Oommen, and D. Nussbaum, "Large-scale neuro-modeling for understanding and explaining some brainrelated chaotic behavior," Simulation-Transactions of the Society for Modeling and Simulation International, vol. 88, no. 11, pp. 1316-1337, 2012.

[23] F. Wendling, K. Ansari-Asl, F. Bartolomei, and L. Senhadji, "From EEG signals to brain connectivity: a model-based evaluation of interdependence measures," Journal of Neuroscience Methods, vol. 183, no. 1, pp. 9-18, 2009.

[24] H. Y. Huang and P. C. Lo, "EEG nonlinear interdependence measure of brain interactions under zen meditation," Journal of Biomedical Engineering Research, vol. 29, no. 4, pp. 286-294, 2008.

[25] M. Breakspear and J. R. Terry, "Detection and description of non-linear interdependence in normal multichannel human EEG data," Clinical Neurophysiology, vol. 113, no. 5, pp. 735-753, 2002.

[26] M. Breakspear and J. R. Terry, "Topographic organization of nonlinear interdependence in multichannel human EEG," NeuroImage, vol. 16, no. 3, pp. 822-835, 2002.

[27] C. J. Stam, M. Breakspear, A.-M. van Cappellen van Walsum, and B. W. van Dijk, "Nonlinear synchronization in EEG and whole-head MEG recordings of healthy subjects," Human Brain Mapping, vol. 19, no. 2, pp. 63-78, 2003.

[28] U. Feldmann and J. Bhattacharya, "Predictability improvement as an asymmetrical measure of interdependence in bivariate time series," International Journal of Bifurcation and Chaos, vol. 14, no. 2, pp. 505-514, 2004.

[29] M. Rubinov, S. A. Knock, C. J. Stam et al., "Small-world properties of nonlinear brain activity in schizophrenia," Human Brain Mapping, vol. 30, no. 2, pp. 403-416, 2009.

[30] P. Mirowski, D. Madhavan, Y. LeCun, and R. Kuzniecky, "Classification of patterns of EEG synchronization for seizure prediction," Clinical Neurophysiology, vol. 120, no. 11, pp. 19271940, 2009.

[31] S. I. Dimitriadis, N. A. Laskaris, Y. del Rio-Portilla, and G. C. Koudounis, "Characterizing dynamic functional connectivity across sleep stages from EEG," Brain Topography, vol. 22, no. 2, pp. 119-133, 2009.

[32] K. Sibsambhu and R. Aurobinda, "Effect of sleep deprivation on functional connectivity of EEG channels," IEEE Transcations on Systems, Man, and Cybernetics, vol. 43, no. 3, pp. 666-672, 2013.

[33] C. M. W. J. M. Tian, Chan Master Miao Tian's Book of Wisdom and the Guide to Heart Chan Meditation, Lulu, 2010.

[34] X.-S. Zhang, R. J. Roy, and E. W. Jensen, "EEG complexity as a measure of depth of anesthesia for patients," IEEE Transactions on Biomedical Engineering, vol. 48, no. 12, pp. 1424-1433, 2001.

[35] I. Daubechies, Ten Lectures on Wavelets, Society for Industrial and Applied Mathematics, Philadelphia, Pa, USA, 1992.

[36] C. Heil, D. F. Walnut, and I. Daubechies, Fundamental Papers in Wavelet Theory, Princeton University Press, Princeton, NJ, USA, 2006.
[37] C. Y. Liu and P. C. Lo, "Spatial focalization of zen-meditation brain based on EEG," Journal of Biomedical Engineering Research, vol. 29, pp. 17-24, 2008.

[38] H. Adeli, Z. Zhou, and N. Dadmehr, "Analysis of EEG records in an epileptic patient using wavelet transform," Journal of Neuroscience Methods, vol. 123, no. 1, pp. 69-87, 2003.

[39] F. Takens, "Detecting strange attractors in turbulence," in Dynamical Systems and Turbulence, D. A. Rand and L. S. Young, Eds., vol. 898 of Lecture Notes in Mathematics, pp. 366-381, Springer, New York, NY, USA, 1981.

[40] P.-C. Lo and W.-P. Chung, "An approach to quantifying the multi-channel EEG spatial-temporal feature," Biometrical Journal, vol. 42, no. 7, pp. 901-916, 2000.

[41] W. S. Pritchard and D. W. Duke, "Dimensional analysis of notask human EEG using the Grassberger-Procaccia method," Psychophysiology, vol. 29, no. 2, pp. 182-192, 1992.

[42] R. Q. Quiroga, A. Kraskov, T. Kreuz, and P. Grassberger, "Performance of different synchronization measures in real data: a case study on electroencephalographic signals," Physical Review E, vol. 65, no. 4, Article ID 041903, 14 pages, 2002. 


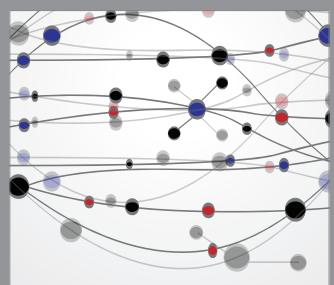

The Scientific World Journal
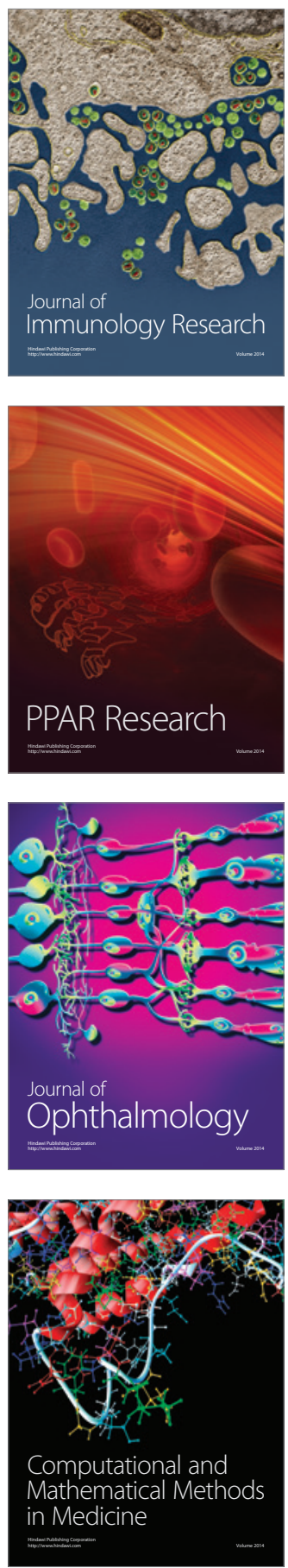

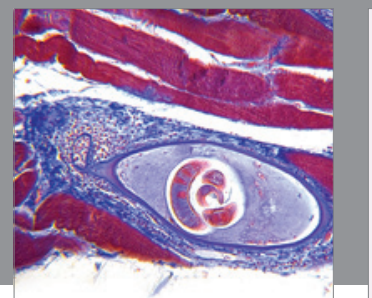

Gastroenterology

Research and Practice
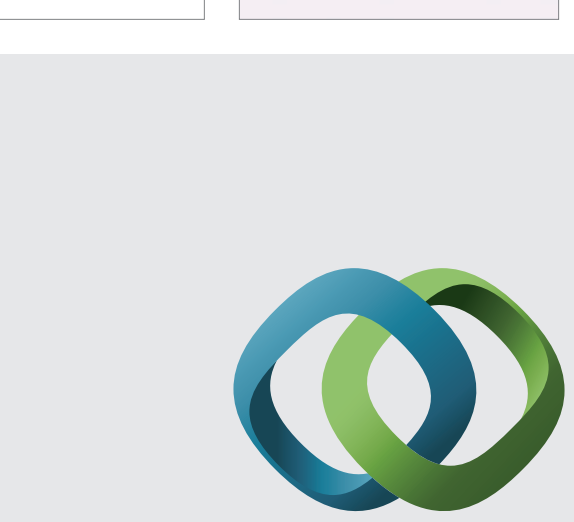

\section{Hindawi}

Submit your manuscripts at

http://www.hindawi.com
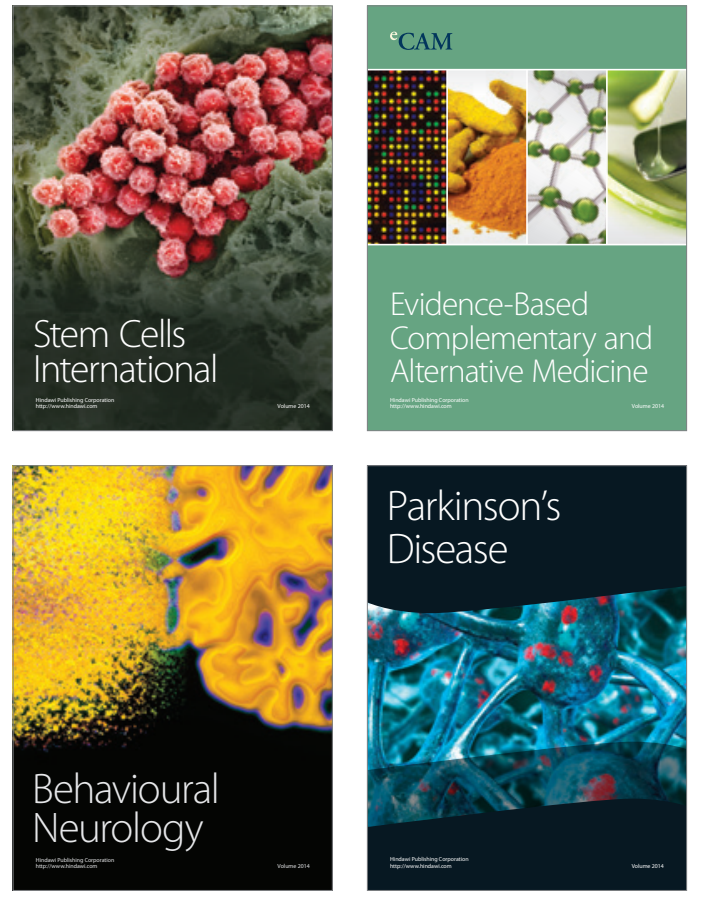
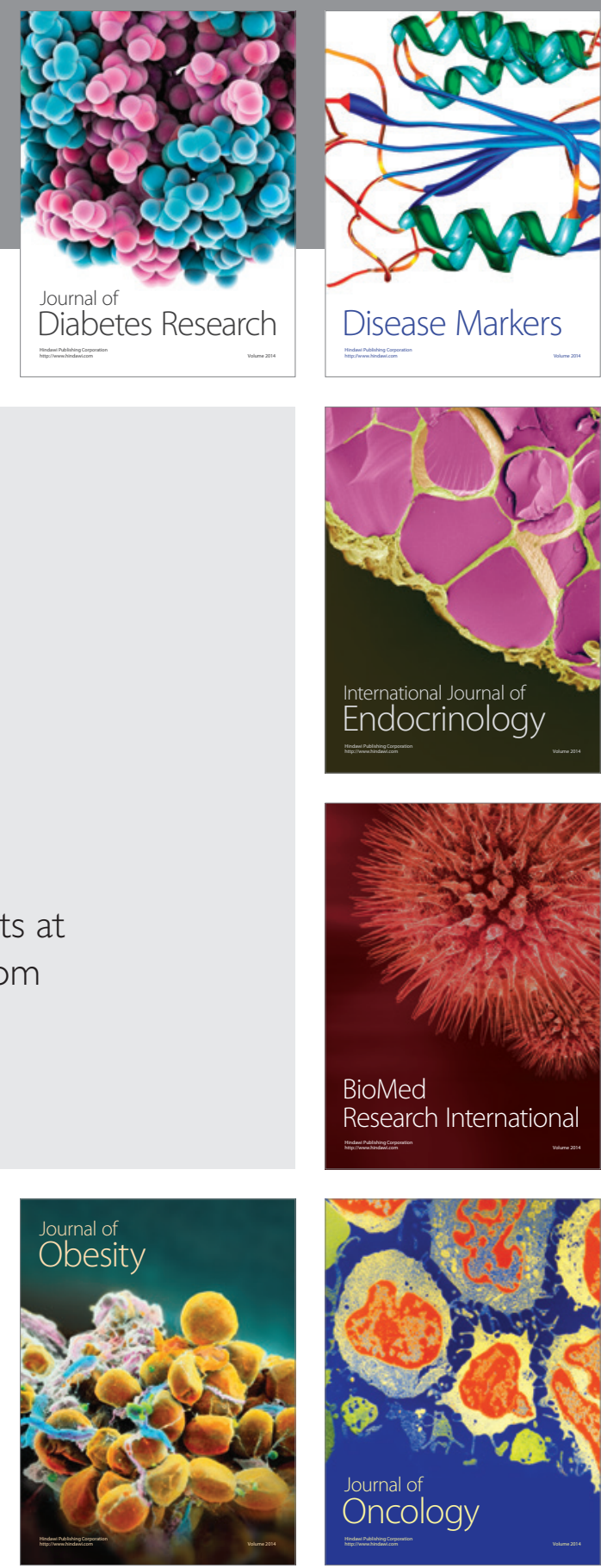

Disease Markers
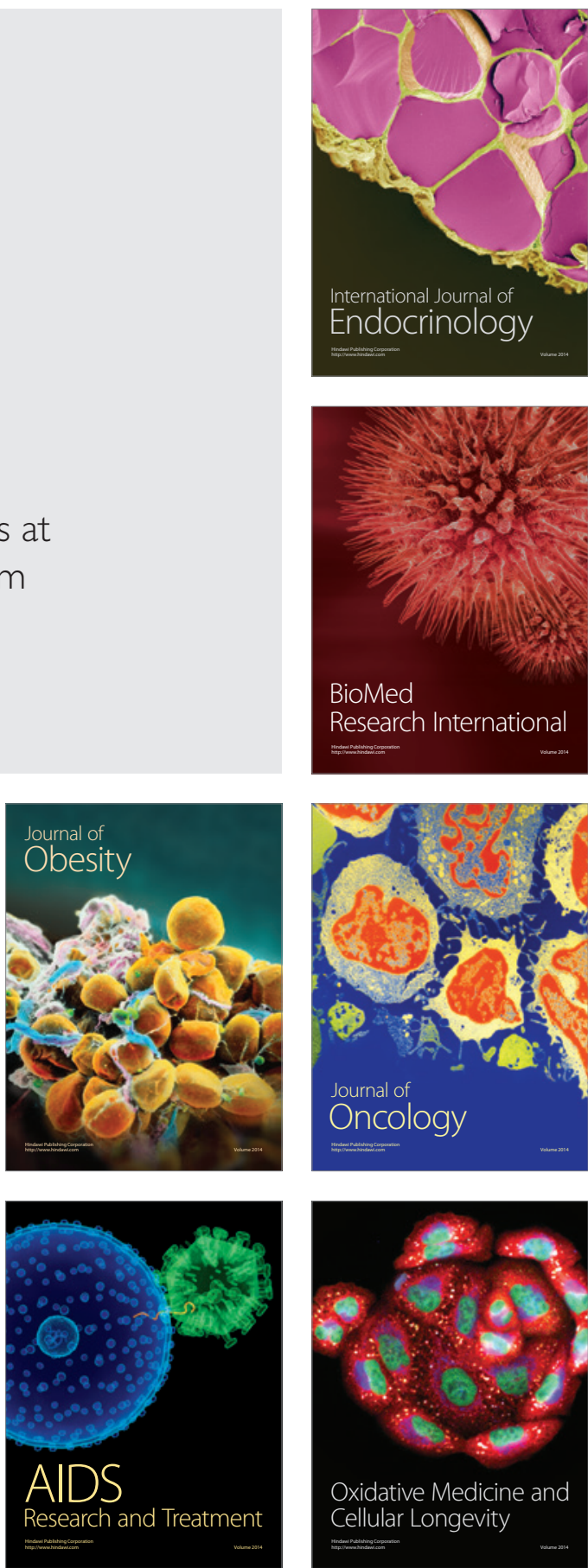